\title{
Discontinuous fuzzy Fredholm integral equations and strong fuzzy Henstock integrals
}

\author{
Yabin Shao ${ }^{1,2}$, Guoliang Xue ${ }^{1}$ \\ 1. College of Mathematics and Computer Science, Northwest University for Nationalities, Lanzhou, China. 2. College of \\ Mathematics, Sichuan University, Chengdu, China \\ Correspondence: Yabin Shao. Address: College of Mathematics and Computer Science, Northwest University for \\ Nationalities, Lanzhou 730030, China. Email: yb-shao@163.com. \\ Received: June 22,2012 \\ Accepted: September 26, 2012 \\ Online Published: February 18, 2013 \\ DOI : $10.5430 /$ air.v2n2p87 \\ URL: http://dx.doi.org/10.5430/air.v2n2p87
}

\section{Abstract}

By using the properties of strong fuzzy Henstock integrals, the existence theorems of solution for a kind of the discontinuous fuzzy Fredholm integral equations are established. The results are generalizations of earlier investigation for fuzzy continuous systems.

\section{Key words}

Fuzzy number, Fuzzy Fredholm integral equation, Existence of solution, Strong fuzzy Henstock integrals

\section{I ntroduction}

To introduce the problem which is considered in this paper, let us consider the general discontinuous fuzzy system defined by the following initial value problem of a fuzzy Fredholm integral equation.

$$
x(t)=f(t)+\int_{0}^{a} k_{1}(t, s) x(s) d s+\int_{0}^{a} k_{2}(t, s) g(x, x(t)) d s, t \in I_{\alpha}=[0, a], a \in R^{+}
$$

Where $f, g, x$ are fuzzy-number-valued functions with some discontinuity and integrals are taken in the sense of strong fuzzy Henstock integrals. Because there are discontinuous systems in which the $f, g, x$ are not integrable in the sense of Kaleva, our results are generalizations of earlier investigation for fuzzy continuous systems.

It is well-known that the Henstock integral includes the Riemann, improper Riemann, Lebesgue and Newton integrals. Though such an integral was defined by Denjoy in 1912 and also by Perron in 1914, it was difficult to handle using their definitions. But with the Riemann-type definition introduced more recently by Henstock ${ }^{[1]}$ in 1963 and also independently by Kurzweil ${ }^{[2]}$, the definition is now simple and furthermore the proof involving the integral also turns out to be easy. For more detailed results about the Henstock integral, we refer to ${ }^{[3]}$. Since the concept of fuzzy sets was first introduced by Zadeh in 1965, it has been studied extensively from many different aspects of the theory and applications, especially in information science, such as linguistic information system and approximate reasoning, fuzzy topology, fuzzy analysis, fuzzy decision making, fuzzy logic and so on. It is well known that the theory of fuzzy sets provides an effective means of 
describing the behavior of system which are too complex or too ill-defined to admit precise mathematical analysis by classical methods and tools. Recently, Wu and Gong ${ }^{[4]}$ have combined the above theories and discussed the fuzzy Henstock integrals of fuzzy-number-valued functions which extended Kaleva integration. In order to complete the theory of fuzzy calculus and to meet the solving need of transferring a fuzzy differential equation into a fuzzy integral equation, we ${ }^{[5,6]}$ have defined the strong fuzzy Henstock integrals and discussed some of their properties and the controlled convergence theorems.

The Cauchy problems for fuzzy differential equations have been studied by several authors ${ }^{[7-10]}$ on the metric space of normal fuzzy convex set with the distance $D$ given by the maximum of the Hausdorff distance between the corresponding level sets. In 2002, Xue and $\mathrm{Fu}^{[11]}$ established solutions to fuzzy differential equations with right-hand side functions satisfying Caratheodory conditions on a class of Lipschitz fuzzy sets.

In this paper, on the existence of the solutions of fuzzy integral equations the Ascoli' s theorem or metric fixed point theorems are used. For the existence and uniqueness, the main tool is the Banach fixed point principle. Such results can be found ${ }^{[12-15]}$. Our fundamental tools are the Kuratowski measures of noncompactess and Monch fixed point theorem ${ }^{[16]}$, and the controlled convergence theorem of strong fuzzy Henstock integrals.

For any bounded subset $A$ of a Banach space $E$ we denote by $\alpha(A)$ the Kuratowski measures of noncompactess of $A$ i.e. the infimun of all $\varepsilon>0$ such that there exist a finite covering of $A$ by set of diameter smaller than $\varepsilon$. We denoted $\alpha$.

We will apply the following fixed point theorem.

Theorem 1.1 ${ }^{[16]}$ Let $D$ be a closed convex subset of a Banach space $E$, and let $F$ be a continuous map from $D$ into itself. If for some $x \in D$ the implication

$$
\bar{V}=\overline{\operatorname{con}}(\{x\} \cup F(V)) \Rightarrow V
$$

is relatively compact, holds for every countable subset $V$ of $D$, then $F$ has a fixed point.

\section{Preliminaries}

\subsection{Fuzzy numbers and fuzzy number space}

Let $P_{k}\left(R^{n}\right)$ denote the family of all nonempty compact convex subset of $R^{n}$ and define the addition and scalar multiplication in $P_{k}\left(R^{n}\right)$ as usual. Let $A$ and $B$ be two nonempty bounded subset of $R^{n}$. The distance between $A$ and $B$ is defined by the Hausdorff metric ${ }^{[7]}$ :

$$
d_{H}(A, B)=\max \left\{\operatorname{supinf}_{a \in A}|a-b|, \operatorname{supinf}_{b \in B}|b-a|\right\} .
$$

Denote $E^{n}=\left\{u: R^{n} \rightarrow[0,1], u\right.$ satisfies (1) - (4) below $\}$ is a fuzzy number space. Where

(1) $u$ is normal, i.e. there exists an $x_{0} \in R^{n}$ such that $u\left(x_{0}\right)=1$; 
(2) $u$ is fuzzy convex, i.e. $u(\lambda x+(1-\lambda) y) \geq \min \{u(x), u(y)\}$ for any $x, y \in R^{n}$ and $0 \leq \lambda \leq 1$;

(3) $u$ is upper semi-continuous;

(4) $[u]^{0}=c l\left\{x \in R^{n} \mid u(x)>0\right\}$ is compact.

Define $D: E^{n} \times E^{n} \rightarrow(0,+\infty)$

$$
D(u, v)=\sup \left\{d_{H}\left([u]^{\alpha},[v]^{\alpha}\right): \alpha \in[0,1]\right\}
$$

where $d_{H}$ is the Hausdorff metric defined in $P_{k}\left(R^{n}\right)$. Then it is easy see that $D$ is a metric in $E^{n}$. Using the results ${ }^{[7]}$, we know that the metric space $\left(E^{n}, D\right)$ has a linear structure, it can imbedded isomorphically as a cone in a Banach space of function $u^{*}: I \times S^{n-1} \rightarrow R$, where $S^{n-1}$ is the unit sphere in $R^{n}$, which an imbedding function $u^{*}=j(u)$ defined by $u^{*}(r, x)=\sup _{\alpha \in[u]^{\alpha}}\langle\alpha, x\rangle$.

It is well know that the $H$-derivative for fuzzy functions was initially introduced by Puri and Ralescu ${ }^{[7]}$ and it is based in the condition $(H)$ of sets. In this paper, we consider a more general definition of a derivative for fuzzy number valued functions enlarging the class of differentiable fuzzy number valued functions, which has been introduced ${ }^{[17]}$.

Definition 2.1 . $^{[17]}$. Let $\tilde{f}:(a, b) \rightarrow E^{n}$ and $x_{0} \in(a, b)$. We say that $\tilde{f}$ is differentiable at $x_{0}$, if there exist an element $\tilde{f}^{\prime}\left(t_{0}\right) \in E^{n}$, such that

(1) For all $h>0$ sufficiently small, there exist $\tilde{f}\left(x_{0}+h\right)-{ }_{H} \tilde{f}\left(x_{0}\right)$ and $\tilde{f}\left(x_{0}+h\right)-{ }_{H} \tilde{f}\left(x_{0}\right)$, the limits

$$
\lim _{h \rightarrow 0} \frac{\tilde{f}\left(x_{0}+h\right)-{ }_{H} \tilde{f}\left(x_{0}\right)}{h}=\lim _{h \rightarrow 0} \frac{\tilde{f}\left(x_{0}\right)-{ }_{H} \tilde{f}\left(x_{0}-h\right)}{h}=\tilde{f}^{\prime}\left(x_{0}\right)
$$

(2) For all $h>0$ sufficiently small, there exist $\tilde{f}\left(x_{0}\right)-{ }_{H} \tilde{f}\left(x_{0}+h\right)$ and $\tilde{f}\left(x_{0}-h\right)-{ }_{H} \tilde{f}\left(x_{0}\right)$, the limits

$$
\lim _{h \rightarrow 0} \frac{\tilde{f}\left(x_{0}\right)-{ }_{H} \tilde{f}\left(x_{0}+h\right)}{-h}=\lim _{h \rightarrow 0} \frac{\tilde{f}\left(x_{0}-h\right)-{ }_{H} \tilde{f}\left(x_{0}\right)}{-h}=\tilde{f}^{\prime}\left(x_{0}\right)
$$

(3) For all $h>0$ sufficiently small, there exist $\tilde{f}\left(x_{0}+h\right)-{ }_{H} \tilde{f}\left(x_{0}\right)$ and $\tilde{f}\left(x_{0}-h\right)-{ }_{H} \tilde{f}\left(x_{0}\right)$, the limits

$$
\lim _{h \rightarrow 0} \frac{\tilde{f}\left(x_{0}+h\right)-{ }_{H} \tilde{f}\left(x_{0}\right)}{h}=\lim _{h \rightarrow 0} \frac{\tilde{f}\left(x_{0}-h\right)-{ }_{H} \tilde{f}\left(x_{0}\right)}{-h}=\tilde{f}^{\prime}\left(x_{0}\right)
$$

(4) For all $h>0$ sufficiently small, there exist $\tilde{f}\left(x_{0}\right)-{ }_{H} \tilde{f}\left(x_{0}+h\right)$ and $\tilde{f}\left(x_{0}\right)-{ }_{H} \tilde{f}\left(x_{0}-h\right)$, the limits 


$$
\lim _{h \rightarrow 0} \frac{\tilde{f}\left(x_{0}\right)-{ }_{H} \tilde{f}\left(x_{0}+h\right)}{-h}=\lim _{h \rightarrow 0} \frac{\tilde{f}\left(x_{0}\right)-{ }_{H} \tilde{f}\left(x_{0}-h\right)}{h}=\tilde{f}^{\prime}\left(x_{0}\right)
$$

\subsection{The strong fuzzy Henstock integral}

In this section we give the strong fuzzy Henstock integral in fuzzy number space and we give some properties of this integral.

Definition $2.2^{[1-3]}$. Let $\delta>0$ be a function on $[a, b]$. A division $P=\left\{\left[c_{i}, d_{i}\right] ; s_{i}\right\}$ is said to be $\delta$-fine, if the following conditions are satisfied:

(1) $a=x_{0}<x_{1}<\cdots<x_{n}=b$,

(2) $s_{i} \in\left[c_{i-1}, c_{i}\right] \subset\left(s_{i}-\delta\left(s_{i}\right), s_{i}+\delta\left(s_{i}\right)\right)$.

Definition $2.3^{[5,6]}$. A function $f:[a, b] \rightarrow E^{n}$ is strong fuzzy Henstock integrable on $[a, b]$ ( $f \in S F H\left([a, b], E^{n}\right)$ if there exist a function $F:[a, b] \rightarrow E^{n}$ with the following property: given $\varepsilon>0$ there exist a positive function $\delta$ on $[a, b]$ such that $P=\left\{\left[c_{i}, d_{i}\right] ; s_{i}\right\}$ is a tagged partition of $[a, b]$, then

$$
\sum_{i=1}^{n} D\left(f\left(s_{i}\right)\left(d_{i}-c_{i}\right), F\left(\left[c_{i}, d_{i}\right]\right)\right)<\varepsilon
$$

where $D$ is the Hausdorff metric of the distance between fuzzy numbers.

Definition 2.4 ${ }^{[5,6]}$ Let $f: I_{\alpha} \rightarrow E^{n}$ be a strong fuzzy Henstock integrable on $[a, b]$. Then the function $F(t)=\int_{a}^{t} f(s) d s$, which is defined on subintervals of $[a, b]$ and the integrals is in the sense of strong fuzzy Henstock, is called the primitive of $f$.

Theorem 2.5 ${ }^{[6]}$ Let $f: I_{\alpha} \rightarrow E^{n}$ be a strong fuzzy Henstock integrable on $[a, b]$ and let $F(t)=\int_{a}^{t} f(s) d s$ for each $s \in[a, b]$. Then

(1) $F$ is continuous on $[a, b]$,

(2) $F$ is differentiable almost everywhere on $[a, b]$,

(3) $f$ is measurable.

\section{Main result}

In this section, we will give the existence theorem of problem (1.1). 
For $x \in C\left(I_{\alpha}, E^{n}\right)$, we define the norm of $H(x, 0)=\sup \left\{D(x(t), 0), t \in I_{\alpha}\right\}$, Let $B(p)=\left\{x \in C\left(I_{\alpha}, E^{n}\right): D(X, 0) \leq D(f, 0)+p\right\}, p>0$.

Note that this set is closed and convex.

We define the operator $F: C\left(I_{\alpha}, E^{n}\right) \rightarrow C\left(I_{\alpha}, E^{n}\right)$ by

$$
F(x)(t)=f(t)+\int_{0}^{a} k_{1}(t, s) x(s) d s+\int_{0}^{a} k_{2}(t, s) g(s, x(t)) d s, t \in I_{\alpha}=[0, a], x \in B(p) .
$$

Definition 3.1 ${ }^{[11]}$ A function $f: I_{\alpha} \rightarrow E^{n}$ is a Caratheodory function if for each $x \in E^{n}, f(t, x)$ is measurable in $t \in I_{\alpha}$ and for almost all $t \in I_{\alpha}, f(t, x)$ is continuous with respect to $x$.

Definition 3.2 A continuous function $x: I_{\alpha} \rightarrow E^{n}$ is said to be a solution of the problem (1.1) if it satisfies:

$$
x(t)=f(t)+\int_{0}^{a} k_{1}(t, s) x(s) d s+\int_{0}^{a} k_{2}(t, s) g(x, x(t)) d s, t \in I_{\alpha}=[0, a], a \in R^{+}
$$

Theorem 3.1 Suppose that $V$ is a countable set of $S F H$ integrable functions. Let $F=\left\{\int_{0}^{t} x(s) d s: x \in V, t \in I_{\alpha}\right\}$ be an equicontinuous, equibounded and uniformly $A C G^{*}$ on $I_{\alpha}$. Then $\alpha\left(j \circ \int_{0}^{t} V(s) d s\right) \leq \int_{0}^{t} \alpha(j \circ V(s)) d s, t \in I_{\alpha}$, whenever $\alpha(j \circ V(s)) \leq \varphi(s), \varphi$ is a Lebesgue integralble function and $j$ is the the isometric embedding from $\left(E^{n}, D\right)$ onto its range in the Banach space $X$ and $\alpha$ is the the Kuratowski's measure of non-compactness of $V$.

Proof. Assume that $Z$ is a linear subspace of $E^{n}$. Because the function $x$ is $S F H$ integrable so for each $\varepsilon=\frac{1}{m}>0, m=1,2, \ldots$ there exists a positive function $\delta_{m}$ such that: if $P=\left\{\xi_{i},\left(t_{i-1}, t_{i}\right): 1 \leq i \leq n\right\}$ is a tagged partition of $I_{\alpha}$ subordinate to $\delta_{m}$ then

$$
\begin{gathered}
D\left(\int_{0}^{t} x(s) d s, \sum_{i=1}^{n} x\left(\xi_{i}\right)\left(t_{i}-t_{i-1}\right)\right)<\varepsilon, \\
\left|\int_{0}^{t} d_{H}(x(s), Z) d s-\sum_{i=1}^{n} d_{H}\left(x\left(\xi_{i}\right), Z\right)\left(t_{i}-t_{i-1}\right)\right|<\varepsilon, t \in I_{\alpha}, x \in V .
\end{gathered}
$$

We have

$$
d_{H}\left(\sum_{i=1}^{n} x\left(\xi_{i}\right)\left(t_{i}-t_{i-1}\right), Z\right) \leq \sum_{i=1}^{n} d_{H}\left(x\left(\xi_{i}\right), Z\right)\left(t_{i}-t_{i-1}\right)
$$

then, 


$$
d_{H}\left(\int_{0}^{t} x(s) d s, Z\right) \leq \int_{0}^{t} d_{H}(x(s), Z) d s .
$$

Let $\int_{0}^{t} V(s) d s=\left\{\int_{0}^{t} X_{m}(s) d s: m=1,2, \cdots\right\}$. Since the function $t \rightarrow d_{H}\left(x_{m}(t), E^{n}\right)$ is measurable on $I_{\alpha}$ so the function $t \rightarrow v(t)=\alpha(j \circ V(t))=\lim _{m \rightarrow \infty} d_{H}\left(x_{m}(t), E^{n}\right)$ is measure on $I_{\alpha}$. Moreover we have $v(t) \leq \varphi(t)$ a.e., where $\varphi$ is a Lebesgue integrable function. So, we have

$$
d_{H}\left(\int_{0}^{t} x_{m}(s) d s, E^{n}\right) \leq \int_{0}^{t} d_{H}\left(x_{m}(s), E^{n}\right) d s .
$$

Hence, we get

$$
\lim _{m \rightarrow \infty} d_{H}\left(\int_{0}^{t} x_{m}(s) d s, E^{n}\right) \leq \lim _{m \rightarrow \infty} \int_{0}^{t} d_{H}\left(x_{m}(s), E^{n}\right) d s \leq \int_{0}^{t} \lim _{m \rightarrow \infty} d_{H}\left(x_{m}(t), E^{n}\right) d s .
$$

So,

$$
\alpha\left(j \circ \int_{0}^{t} V(s) d s\right) \leq \int_{0}^{t} \alpha(j \circ V(s)) d s, t \in I_{\alpha} .
$$

The proof is completed.

Theorem 3.2 If the function $f: I_{\alpha} \rightarrow E^{n}$ is $S F H$ integrable, then

$$
\int_{I} f(t) d t \in \mid I \overline{\operatorname{conv}} f(I)
$$

where $I$ is an arbitrary subinterval of $I_{\alpha}$.

Proof. Because $j \circ f(t)$ is Henstock integration, by the mean value theorem for Henstock integral of real valued function, we have

$$
\overline{\operatorname{conv}} j \circ f(I)=|I| \cdot j \circ \overline{\operatorname{conv}} f(I) .
$$

But, by the definition of strong fuzzy Henstock integral, there exists $\int_{I} f(t) d t$ such that $(H) \int_{I} j \circ f(t) d t=j \circ \int_{I} f(t) d t$. So, $j \circ \int_{I} f(t) d t \in|I| \cdot j \circ \overline{\operatorname{conv}} f(I)$ for the imbedding function $j$. Because the set $\{|I| \cdot j \circ \overline{\operatorname{conv}} f(I)\}$ is a closed convex set, this implies $\int_{I} f(t) d t \in|I| \overline{\operatorname{conv}} f(I)$.

Theorem 3.3 Assume that for each continuous function $x: I_{\alpha} \rightarrow E^{n}, g(\cdot, x(\cdot))$ is $S F H$ integrable and $g$ is a Caratheodory function. Let $k_{1}, k_{2}: I_{\alpha} \times I_{\alpha} \rightarrow R^{+}$be measurable functions such that $k_{1}(t, \cdot), k_{2}(t, \cdot)$ are continuous. Moreover, let there exists $p_{0}>0$ and a Caratheodory function $\omega: I_{\alpha} \times R^{+} \rightarrow R^{+}$with 


$$
\alpha(j \circ g(s, X)) \leq \omega(s, \alpha(j \circ X)) \text { for a.e. } s \in I_{\alpha} \text { and } X \subset B(p) \text {, }
$$

such that the zero function is the unique solution of the inequality

$$
q(t) \leq 2\left[\int_{0}^{c} k_{1}(t, s) q(s) d s+\int_{0}^{c} k_{2}(t, s) \omega(s, q(s)) d s\right]
$$

Suppose that $\Gamma\left(p_{0}\right)$ is equicontinuous, equibounded and uniformly $A C G^{*}$ on $I_{\alpha}$. Then there exist at least solution of the problem (1.1) on $I_{c}$ for some $0<c \leq a$ with continuous initial function $f$.

Proof. By equicontinuty and equibounded of $\Gamma\left(p_{0}\right)$ there exist some number $c(0<c \leq a)$, such that $D\left(\int_{0}^{c}\left[k_{1}(t, s) x(s)+k_{2}(t, s) g(s, x(t)] d s, 0\right) \leq p_{0}\right.$, for $t \in I_{c}$ and $x \in B\left(p_{0}\right)$.

By assumption, the operator $F$ is well defined and map $B\left(p_{0}\right)$ to $B\left(p_{0}\right)$. We now show that the operator $F$ is continuous. In fact, the function $g$ is a Caratheodory function, so from inequality

$$
\left.H\left(F\left(x_{n}\right), F(x)\right)=H\left(\int_{0}^{c} k_{1}(t, s)\left(x_{n}(s)-x(s)\right)+k_{2}(t, s)\left(g\left(s, x_{n}(s)\right)-g(s, x(s))\right)\right) d s, 0\right)
$$

we have $F\left(x_{n}\right) \rightarrow F(x)$. Observe that a fixed point of $F$ is the solution of the problem (1.1). Next we prove that $F$ has a fixed point using Theorem 1.1.

In fact, we assume that $V \subset B\left(p_{0}\right)$ satisfies condition $\bar{V}=\overline{\operatorname{con}}(\{x\} \cup F(V))$ for some $x \in B\left(p_{0}\right)$. Let $V(t)=\left\{v(t) \in E^{n}: v \in V\right\}$ for $t \in I_{\alpha}$. Since $V \subset B\left(p_{0}\right), F(V) \subset \Gamma\left(p_{0}\right)$. Then $V \subset \bar{V}=\overline{\operatorname{con}}(\{x\} \cup F(V))$ is equicontinuous.

Let $\int_{0}^{c} Z(s) d s=\left\{\int_{0}^{c} x(s) d s: x \in Z\right\}$ for any $Z \in C\left(I_{c}, E^{n}\right)$ and let $\tilde{g}$ denote the mapping defined by $\tilde{g}(x(s))=g(s, x(s))$ for each $x \in B\left(p_{0}\right)$ and $s \in I_{c}$. Obviously, we have the following $\tilde{g}(V(s))=g(s, V(s))$ and

$$
F(V(t))=f(t)+\int_{0}^{c}\left[k_{1}(t, s) V(s)+k_{2}(t, s) \tilde{g}(V(s))\right] d s
$$

hold ture. Using (3.1) and Theorem 3.1 and the properties of the measure of noncompactness $\alpha^{[16]}$, we have

$$
\begin{gathered}
\alpha(j \circ(F(V)(t)))=\alpha\left(j \circ\left(f(t)+\int_{0}^{c}\left[k_{1}(t, s) V(s)+k_{2}(t, s) \tilde{g}(V(s))\right] d s\right)\right) \leq \\
\left.\leq 2 \alpha\left(j \circ \int_{0}^{c}\left[k_{1}(t, s) V(s)+k_{2}(t, s) \tilde{g}(V(s))\right] d s\right)\right)
\end{gathered}
$$




$$
\begin{gathered}
\leq 2 \int_{0}^{c}\left[k_{1}(t, s) \alpha(j \circ V(s))+k_{2}(t, s) \alpha(j \circ \tilde{g}(V(s))] d s\right. \\
\leq 2 \int_{0}^{c}\left[k_{1}(t, s) \alpha(j \circ V(s))+k_{2}(t, s) \alpha(j \circ g(V(s))] d s\right. \\
\leq 2 \int_{0}^{c}\left[k_{1}(t, s) \alpha(j \circ V(s))+k_{2}(t, s) \omega(s, \alpha(j \circ V(s)))\right] d s
\end{gathered}
$$

Because $\bar{V}=\overline{\operatorname{con}}(\{x\} \cup F(V))$, we have

$$
v(t)=2\left[\int_{0}^{c} k_{1}(t, s) v(s) d s+\int_{0}^{c} k_{2}(t, s) \omega(s, v(s)) d s\right]
$$

By our assumptions, because the zero function is the unique continuous solution of the last inequality, we get $v(t)=\alpha(j \circ V(t))=0$. By Arzela-Asocli's theorem ${ }^{[16]}, V$ is relatively compact. So, by Theorem 1.1, $F$ has a fixed point which is a solution of the problem (1.1). The proof is completed.

\section{Conclusions}

In this paper, we deal with the Cauchy problem of discontinuous fuzzy differential equations involving the strong fuzzy Henstock integral in fuzzy number space. The function governing the equations is supposed to be discontinuous with respect to some variables and satisfy nonabsolute fuzzy integrability. Our result improves the result given ${ }^{[4,6,8,12]}$ (where uniform continuity was required), as well as those referred therein.

\section{Acknowledgements}

This work is supported by the National Natural Scientific Fund of China (No. 11161041, 71061013); Fundamental Research Funds for the Central Universities (No. Zyz2012089).

\section{References}

[1] R. Henstock. Theory of Integration. Butterworth, London; 1963.

[2] J. Kurzweil. Generalized ordinary differential equations and continuous dependence on a parameter. Czechoslovak Mathematical Journal. 1957; 7: 418-46.

[3] P. Lee. Lanzhou Lectures on Henstock Integration. World Scientific, Singapore, New Jersey, London, Hongkong; 1989.

[4] C. Wu, Z. Gong. On Henstock intergrals of interval-valued and fuzzy-number-valued functions. Fuzzy Sets and Systems. 2000; 115: 377-91. http://dx.doi.org/10.1016/S0165-0114(98)00277-2

[5] Z. Gong. On the problem of characterizing derivatives for the fuzzy-valued functions (II). Fuzzy Sets and Systems. 2004; 145: 381-93. http://dx.doi.org/10.1016/S0165-0114(03)00264-1

[6] Z. Gong, Y. Shao. The controlled convergence theorems for the strong Henstock integrals of fuzzy-number-valued functions. Fuzzy Sets and Systems. 2009; 160: 1528-46. http://dx.doi.org/10.1016/j.fss.2008.10.013

[7] Kaleva. Fuzzy differential equations. Fuzzy Sets and Systems. 1987; 24: 301-19. http://dx.doi.org/10.1016/0165-0114(87)90029-7

[8] J. J. Nieto. The Cauchy problem for continuous fuzzy differential equations. Fuzzy Sets and Systems. 1999; 102: $259-62$. http://dx.doi.org/10.1016/S0165-0114(97)00094-8

[9] C. Wu, S. Song, E. Lee. Approximate solutions and existence and uniqueness theorem to the Cauchy problem of fuzzy differential equations. Journal Mathematical Analysis and Applications. 1996; 202: 629-44. http://dx.doi.org/10.1006/jmaa.1996.0338 
[10] Z. Gong, Y. Shao. Global Existence and Uniqueness of solutions for fuzzy differential equations under dissipative-type conditions. Computers \& Mathematics with Applications. 2008; 56: 2716-23. http://dx.doi.org/10.1016/j.camwa.2008.05.029

[11] X. Xue, Y. Fu, Caratheodory solution of fuzzy differential equations. Fuzzy Sets and Systems. 2002; 125: $239-43$. http://dx.doi.org/10.1016/S0165-0114(00)00111-1

[12] Shiji Song, Qinyu Liu, Qichun Xu. Existence and comparison theorems to Volterra fuzzy integral equation in $\left(E^{n}, D\right)$. Fuzzy Sets and Systems. 1999; 104: 315-21. http://dx.doi.org/10.1016/S0165-0114(97)00211-X

[13] Jong Yeoul Park, Jae Ug Jeong. On the existence and uniqueness of solutions of fuzzy Volterra-Fredholm integral equations. Fuzzy Sets and Systems. 2000; 115: 425-31. http://dx.doi.org/10.1016/S0165-0114(98)00341-8

[14] Juan J. Nieto, Rosana Rodríguez-López. Bounded solutions for fuzzy differential and integral equations. Chaos, Solitons \& Fractals. 2006; 27: 1279-92.

[15] Molabahrami, A. Shidfar, A. Ghyasi. An analytical method for solving linear Fredholm fuzzy integral equations of the second kind. Computers \& Mathematics with Applications. 2011; 61: 2754-61. http://dx.doi.org/10.1016/j.camwa.2011.03.034

[16] Lakshmikantham, S.Leela. Nonlinear Differential Equations in Abstract Spaces. Pergamon Press, New York; 1981.

[17] B. Bede, S. Gal. Generalizations of the differentiability of fuzzy-number-valued functions with applications to fuzzy differential equation. Fuzzy Sets and Systems. 2005; 151: 581-99. http://dx.doi.org/10.1016/j.fss.2004.08.001 